\title{
Energy relaxation via confined and interface phonons in quantum-wire systems
}

\author{
C. R. Bennett \\ Department of Physics, University of Essex, Colchester, CO4 3SQ, England \\ B. Tanatar \\ Department of Physics, Bilkent University, Bilkent, 06533 Ankara, Turkey
}

(Received 13 November 1996)

\begin{abstract}
We present a fully dynamical and finite temperature study of the hot-electron momentum relaxation rate and the power loss in a coupled system of electrons and confined and interface phonons in a quantum-wire structure. Renormalization effects due to electron-phonon interactions lead to an enhancement in the power loss similar to the bulk phonon case. [S0163-1829(97)05112-6]
\end{abstract}

\section{INTRODUCTION}

In recent years, the hot-electron energy relaxation phenomenon in low-dimensional semiconductors attracted considerable interest both experimentally ${ }^{1}$ and theoretically. ${ }^{2}$ The energy-loss mechanism is very important because of its technological relevance, as most semiconductor-based devices operate under high-field, hot-electron conditions. In particular, hot-electron transistors with a base region made of high-mobility semiconducting material like GaAs offer a high-speed device. When a strong electric field is applied, the electron gas attains a temperature higher than that of the surrounding lattice. Equilibrium is reached by the emission of different types of phonons depending on the temperature regime. The advances in growth technology made it possible to study quasi-one-dimensional (Q1D) electronic structures, thereby improving our knowledge on low-dimensional systems. Theoretical work on the energy-loss rate in quantum wires has started to appear. ${ }^{3-5}$

In low-dimensional semiconductor structures phonon confinement becomes an essential part of the description of electron-phonon interactions. Since the early observation of confined phonons in GaAs/AlAs superlattices, ${ }^{6}$ the phonon modes in microstructures have attracted a great deal of attention. ${ }^{7,8}$ Among the various macroscopic pictures, the dielectric continuum (DC) model $^{9}$ offers a simple framework with which to address the phonon confinement effects. The phonon modes in the DC model are (i) an infinite set of confined modes with vanishing electrostatic potentials at the interfaces that oscillate at the bulk LO-phonon frequency of GaAs, and (ii) a set of modes with electrostatic potentials attaining maxima at the interfaces. The interface modes lie within the reststrahl band of GaAs and AlAs, and in quasitwo-dimensional (Q2D) systems, it is found ${ }^{10,11}$ that the AlAs interface modes dominate the interaction. The situation is similar in Q1D systems, as demonstrated in the confined and interface polaron problem in cylindrical quantum wires. $^{12}$

The purpose of this paper is to study the energy relaxation via confined and interface phonons of an excited Q1D electron gas in a GaAs quantum wire embedded in AlAs material. We employ the dielectric continuum model to describe the phonon confinement effects and take the many-body renormalization effects due to electron-phonon interactions into account. Thus our work complements the recent study by Zheng and Das Sarma ${ }^{5}$ who considered the energy relaxation by bulk LO phonons. Earlier works taking phonon confinement effects into consideration in quantum wires have neglected the many-body renormalization. ${ }^{13}$ Hot-electron experiments ${ }^{14}$ to date are performed on wide quantum wires with multisubband occupation, but it is conceivable that in the near future quantum-wire structures with only the lowest subband occupied ${ }^{15}$ will be amenable to measurements directly relevant to calculations presented here. The manybody effects change the phonon self-energy (due to electronphonon interactions), renormalizing the phonon propagator significantly at low temperatures.

We use the theory advanced by Das Sarma and co-workers ${ }^{2,5,16}$ to calculate the hot-electron power loss due to confined and interface phonons. A test electron is assumed to be injected into the quantum wire without modifying the properties of the coupled electron-phonon system. The standard electron-scattering theory ${ }^{17}$ is used, treating the system to be not completely isolated. The coupled system is then in quasiequilibrium and interacts with an external heat bath. We note that the above viewpoint was challenged ${ }^{18}$ predicting differing results. Nevertheless, the electron-temperature model $^{2,5,16}$ provides a suitable scheme to describe the energy relaxation processes especially when hot-phonon effects are not important.

The rest of this paper is organized as follows. In the next section, we introduce the expressions for momentum relaxation rate and power loss in Q1D wires. Our numerical results for GaAs quantum wires embedded in AlAs material are presented in Sec. III. We conclude with a brief summary in Sec. IV.

\section{THEORY}

The quantum-wire model we use consists of an infinitely long cylinder of radius $R$ with hard walls. ${ }^{19}$ Such a model leads to an analytic expression ${ }^{19}$ for the effective Coulomb potential $V(q)$ between the electrons within certain approximations. We assume that the linear electron density is such that only the lowest subband is populated. To describe the phonon confinement, we consider a GaAs quantum wire em- 

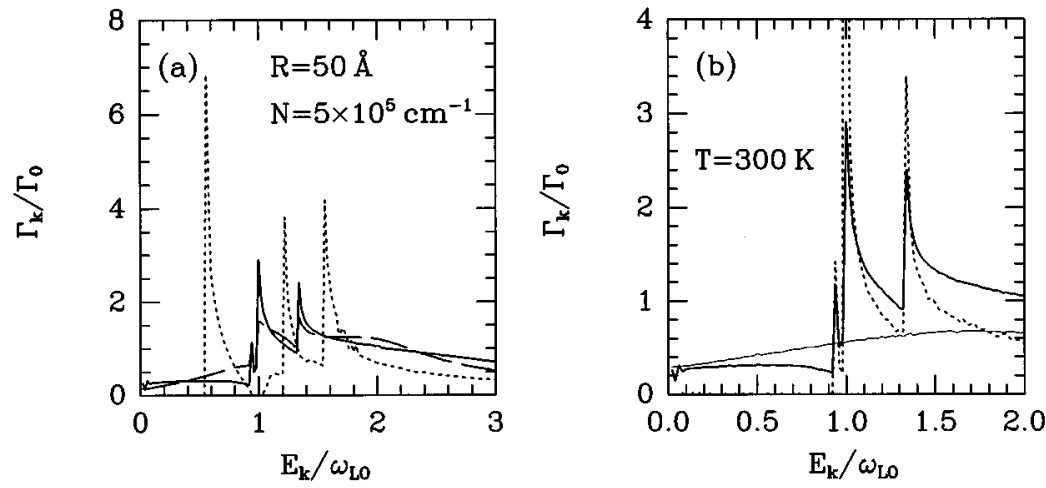

FIG. 1. (a) The momentum relaxation rate of a test electron via confined and interface phonons in a quantum wire of radius $R=50 \AA$, and $N=5 \times 10^{5} \mathrm{~cm}^{-1}$ at $T=0$ (dotted), $T=100 \mathrm{~K}$ (dashed), and $T=300 \mathrm{~K}$ (solid), within the dynamical screening approach. (b) The momentum relaxation rate at $T=300 \mathrm{~K}$ [the rest of the parameters are same as in (a)] in the dynamically screened (solid) and unscreened (dotted) approximations. The thin solid line gives the scattering due solely to uncoupled plasmons. $\Gamma_{0}=8.7$ $\mathrm{ps}^{-1}$.

bedded in AlAs material. Working within the dielectric continuum model we have the confined LO-phonon modes inside the GaAs wire, and the interface phonon modes at the boundary. Scattering rates for embedded wires using the DC model were calculated by several researchers. ${ }^{20}$ Analytic expressions with the approximate electronic wave functions were obtained by Bennett et al. ${ }^{21}$

In this work, we are mainly interested in the relaxation processes via confined and interface phonons. To this end, we first calculate the momentum relaxation rate given by ${ }^{22}$

$$
\begin{aligned}
\Gamma_{k}= & \frac{2}{\hbar} \sum_{q} \frac{q}{k} V(q)\left[n\left(\omega_{k q}\right)+1\right]\left[1-f\left(E_{k-q}\right)\right] \\
& \times \operatorname{Im}\left[-\frac{1}{\varepsilon_{T}\left(q, \omega_{k q}\right)}\right],
\end{aligned}
$$

where $\hbar \omega_{k q}=E_{k}-E_{k-q}, E_{k}=\hbar^{2} k^{2} / 2 m^{*}$, and $n(\omega)$ and $f\left(E_{k}\right)$ are Bose and Fermi distribution functions, respectively. The total dielectric function consists of polarizations from the electron gas (within the RPA) and the phonon part, which is given by

$$
\varepsilon_{T}(q, \omega)=\left[1+\frac{V_{\mathrm{ph}}(q, \omega)}{V(q)}\right]^{-1}-V(q) \chi_{0}(q, \omega),
$$

where $\chi_{0}$ is the noninteracting polarization function for a 1D electron gas, and $V_{\mathrm{ph}}$ is the potential due to various phonon modes

$$
V_{\mathrm{ph}}(q, \omega)=\sum_{i}\left|M_{i}(q)\right|^{2} D_{i}(q, \omega)
$$

Here $M_{i}$ is the matrix element of the electron-phonon interaction in the ground state, and $D_{i}$ is the phonon propagator (Green's function)

$$
D(q, \omega)=\frac{2}{\hbar} \frac{\omega_{i q}}{\omega^{2}-\omega_{i q}^{2}},
$$

for the $i$ th phonon mode with dispersion $\omega_{i q}$. For bulk LO phonons one has $|M(q)|^{2}=V(q)\left(1-\epsilon_{\infty} / \epsilon_{s}\right) \hbar \omega_{\mathrm{LO}} / 2$, which yields the usual coupled-mode dielectric function

$$
\varepsilon_{T}(q, \omega)=1+\frac{\omega_{\mathrm{LO}}^{2}-\omega_{\mathrm{TO}}^{2}}{\omega_{\mathrm{TO}}^{2}-\omega^{2}}-V(q) \chi_{0}(q, \omega) .
$$

Within the DC model, the phonon potentials for confined and interface phonon modes in a wire with circular cross section are expressed as $^{21}$

$$
\begin{aligned}
& V_{C}(q, \omega)= \sum_{j} \frac{e^{2}}{\epsilon_{0} V_{0} J_{1}^{2}\left(\alpha_{0 j}\right)\left(q^{2}+\alpha_{0 j}^{2} / R^{2}\right)}\left(\frac{1}{\epsilon_{\infty}}-\frac{1}{\epsilon_{s}}\right) \\
& \times\left[48 \frac{J_{3}\left(\alpha_{0 j}\right)}{\alpha_{0 j}^{3}}\right]^{2} \frac{\omega_{\mathrm{LO}}}{\omega^{2}-\omega_{\mathrm{LO}}^{2}}, \\
& V_{I F}(q, \omega)= \sum_{j} \frac{e^{2} \epsilon_{2}\left(\omega_{0 j q}\right) R}{q \epsilon_{0} V_{0} I_{0}(q R) I_{1}(q R) D\left(\omega_{0 j q}\right)} \\
& \times\left[48 \frac{I_{3}(q R)}{(q R)^{3}}\right]^{2} \frac{\omega_{0 j q}}{\omega^{2}-\omega_{0 j q}^{2}} .
\end{aligned}
$$

Here $J_{n}(x)$ and $I_{n}(x)$ are Bessel functions, $\alpha_{0 j}$ is the $j$ th root of $J_{0}(x)$,
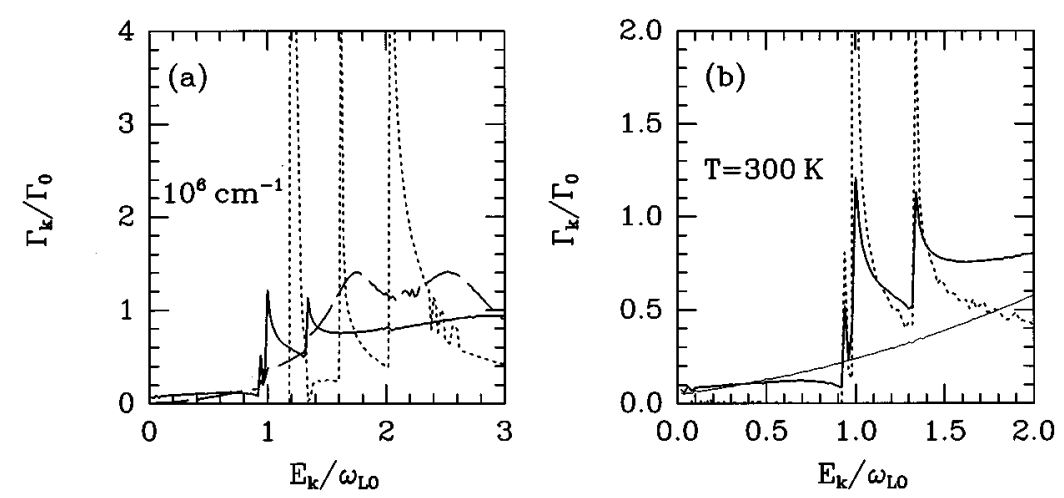

FIG. 2. The same as in Fig. 1 except the density of the electron gas is $N=10^{6} \mathrm{~cm}^{-1}$. 

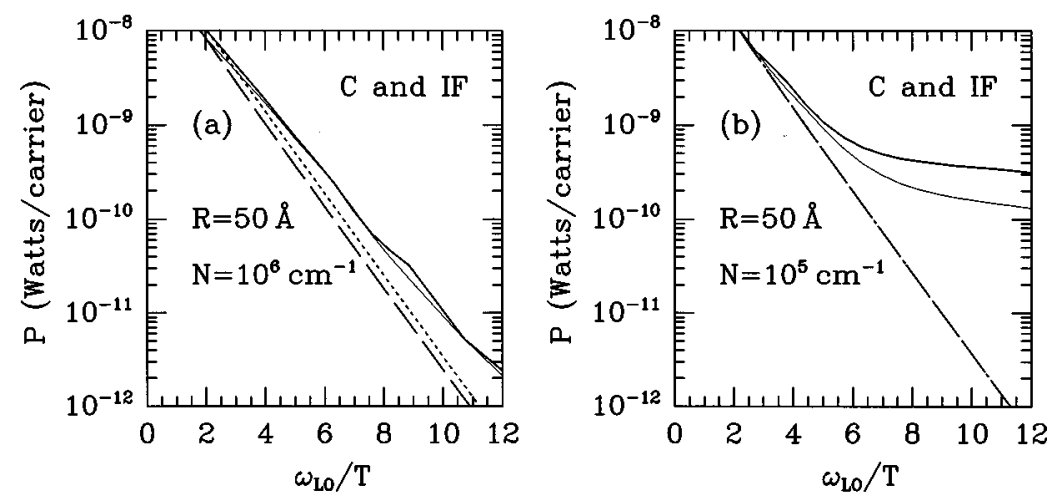

FIG. 3. (a) Power loss per carrier via DC phonon modes as a function of inverse temperature for a $R=50 \AA$, and $N=10^{6} \mathrm{~cm}^{-1} \mathrm{GaAs}$ quantum wire embedded in AlAs material. Solid, dashed, and dotted lines indicate dynamically screened, uncoupled screened, and unscreened approximations, respectively. Thin solid line gives the result for bulk GaAs LO phonons. (b) Same as in (a) at $N=10^{5} \mathrm{~cm}^{-1}$.

$$
D(\omega)=\epsilon_{2}(\omega) \frac{\partial \epsilon_{1}(\omega)}{\partial \omega}-\epsilon_{1}(\omega) \frac{\partial \epsilon_{2}(\omega)}{\partial \omega}
$$

and $\epsilon_{1,2}(\omega)$ are the (1) GaAs and (2) AlAs phonon dielectric functions given by

$$
\epsilon(\omega)=\epsilon_{\infty} \frac{\omega^{2}-\omega_{\mathrm{LO}}^{2}}{\omega^{2}-\omega_{\mathrm{TO}}^{2}} .
$$

The confined phonons have the GaAs zone center frequency while the interface modes have frequencies that lie in the reststrahl band of the wire and barrier materials. Only the lowest-order confined and interface modes interact in a onesubband approximation. A more complete description of DC phonon modes interacting with Q1D electrons is given by Bennett et al. ${ }^{21}$

The next quantity we wish to evaluate is the power loss formula. We assume that the lattice temperature is much smaller than the electron temperature, viz., $T_{L}=0$. The power loss (or energy relaxation rate) within the electron temperature model is given by ${ }^{2,5,17}$

$$
\begin{aligned}
P= & \frac{2}{\pi \hbar} \sum_{q} \int_{0}^{\infty} d(\hbar \omega) \hbar \omega n_{T}(\omega) \operatorname{Im}\left[\chi_{0}(q, \omega)\right] \\
& \times \operatorname{Im}\left[V_{\mathrm{sc}-\mathrm{ph}}(q, \omega)\right],
\end{aligned}
$$

in which $T$ is the electron temperature and the screened phonon potential is

$$
V_{\mathrm{sc}-\mathrm{ph}}(q, \omega)=V(q)\left[\frac{1}{\varepsilon_{T}(q, \omega)}-\frac{1}{\varepsilon(q, \omega)}\right],
$$

where $\varepsilon(q, \omega)=1-V(q) \chi_{0}(q, \omega)$ is the dielectric function of the electronic system only. $V_{\text {sc-ph }}$ is the potential due only to phonon modes. Another way of writing this is

$$
V_{\mathrm{sc}-\mathrm{ph}}(q, \omega)=\frac{V_{\mathrm{ph}}(q, \omega)}{\varepsilon^{2}(q, \omega)\left[1-V_{\mathrm{ph}}(q, \omega) \chi_{0}(q, \omega) / \varepsilon(q, \omega)\right]},
$$

which for the case of one type of phonon leads to

$$
V_{\mathrm{sc}-\mathrm{ph}}=\frac{|M|^{2}}{\varepsilon^{2}(q, \omega)} D^{\prime}(q, \omega)
$$

with the identification of a renormalized phonon propagator

$$
D^{\prime}(q, \omega)=\frac{2 \omega_{q}}{\hbar\left(\omega^{2}-\omega_{q}^{2}\right)-2 \omega_{q}|M|^{2} \chi_{0}(q, \omega) / \varepsilon(q, \omega)} .
$$

When dealing with confined and interface modes within the DC model, the above formulation is not advantageous. For bulk LO phonons, if the many-body coupling is ignored one obtains $\quad D(q, \omega)=\pi\left[\delta\left(\omega+\omega_{\mathrm{LO}}\right)-\delta\left(\omega-\omega_{\mathrm{LO}}\right)\right]$, which gives the uncoupled screened power loss [not static since $\omega=\omega_{\mathrm{LO}}$ in the dielectric function $\left.\varepsilon(q, \omega)\right]$ via bulk LOphonon modes,

$$
P=\frac{2}{\hbar} \sum_{q} \hbar \omega_{\mathrm{LO}} n_{T}\left(\omega_{\mathrm{LO}}\right) \operatorname{Im} \chi_{0}\left(q, \omega_{\mathrm{LO}}\right) \frac{|M|^{2}}{\varepsilon^{2}\left(q, \omega_{\mathrm{LO}}\right)} .
$$

Putting $\varepsilon^{2}=1$ reduces this to the unscreened power loss formula via uncoupled phonons. Similar expressions are found for confined and interface modes.

\section{RESULTS AND DISCUSSION}

We now present our results on the momentum relaxation rates $\Gamma_{k}$ and power loss $P$, via confined and interface phonon modes, in the DC model. We calculate $P$ in three different approximations: the unscreened case when the dielectric function in Eq. (14) is set equal to unity, the uncoupled screened case, when the many-body renormalization is not included but $\varepsilon^{2}\left(q, \omega_{\mathrm{LO}}\right)$ is retained, and finally the dynamically screened case, which includes the many-body renor-

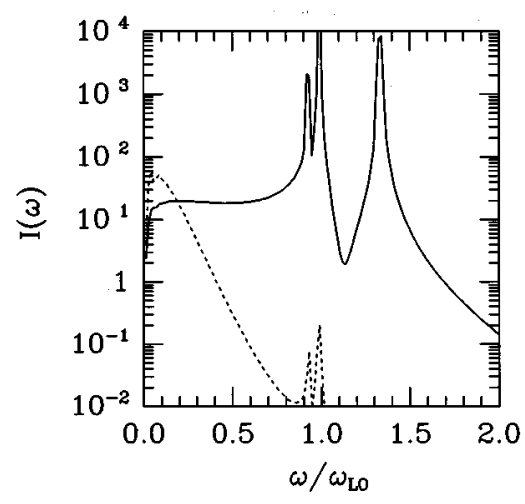

FIG. 4. Power loss spectrum $I(\omega)$ for DC phonon modes in a $R=50 \AA, N=10^{5} \mathrm{~cm}^{-1}$ quantum wire at $T=300 \mathrm{~K}$ (solid line), and $T=30 \mathrm{~K}$ (dotted line). 

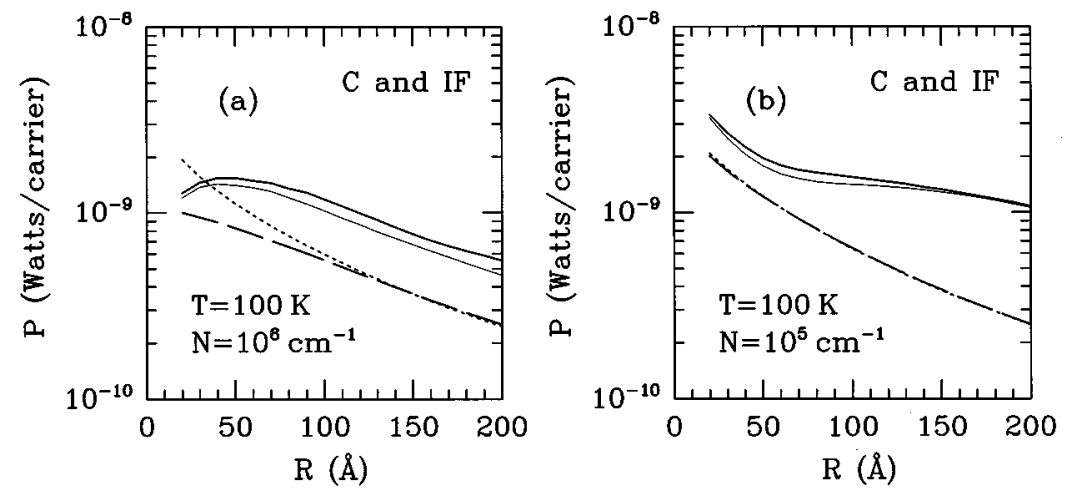

FIG. 5. (a) Power loss per carrier via DC phonon modes as a function of quantum wire radius, at $T=100 \mathrm{~K}$ and $N=10^{6} \mathrm{~cm}^{-1}$. Solid, dashed, and dotted lines indicate dynamically screened, uncoupled screened, and unscreened approximations, respectively. Thin solid line gives the result for bulk GaAs LO phonons. (b) Same as in (a) at $N=10^{5} \mathrm{~cm}^{-1}$.

malization along with the full frequency dependence of $\varepsilon(q, \omega)$. In the numerical calculations we use material parameters appropriate for GaAs and AlAs as given by Adachi. $^{23}$

Figures 1 and 2 show the momentum relaxation rates for coupled DC confined and interface phonon plasmons. At low temperatures three sharp peaks are observed, which are due to the onset of different modes and follow the form of the one-dimensional density of states, $\sim E^{-1 / 2}$. The lower of these is the "plasmonlike" mode, while the others are due to the confined and interface "phononlike" modes. As the temperature increases the coupling is reduced as the plasmon mode becomes damped by single-particle excitations. This reduces the sharp onsets of the peaks until the phonon modes become sufficiently uncoupled to produce their usual interaction with the electrons. In Figs. 1(b) and 2(b) a comparison is made between the uncoupled interactions and the coupled DC phonon plasmons. It can be seen that a sum of the rates due to confined and interface phonons and the plasmons is approximately equal to the coupled mode result. It should be noted that, even at higher temperatures where the phonons produce the largest rates, the plasmon scattering is important and the coupling vital for low temperatures. Hence, just including the electron gas by a static screening term is not sufficient.

Figure 3 shows the power loss via DC confined and interface modes. The gradient indicates the energy of the mode that is emitted. For temperatures about or below room temperature this is still the GaAs phonon energy for the uncoupled DC phonons; the temperature is too low for the emission of the AlAs interface modes and hence the main contribution is from the GaAs confined modes. The uncoupled screened power loss has the same gradient and is lower as the density of the electrons is increased. This is not surprising since the energy of the modes emitted has not changed, and the greater the electron density the larger the effect of screening on reducing the phonon potentials will be.

The power loss via coupled modes is much higher at low temperatures than the uncoupled case and it can be seen that the gradient has reduced. This suggests that the energy of the emitted mode has decreased and this has also been seen for bulk LO phonon plasmons by Zheng and Das Sarma. ${ }^{5}$ Figure 4 shows the frequency integrand of the power loss and it can be seen that at high temperatures it is the GaAs confined modes that dominate while at low temperatures it is modes with a lower energy, produced by the coupling with the electron gas, that are emitted.
Figure 3 also shows the power loss due to coupled bulk LO phonon plasmons. The approximate sum rule, which states that the DC phonons should give a result similar to the the bulk modes of either material, appears to hold here too. This is shown further in Fig. 5. For small radii the power loss should reduce to the AlAs bulk phonon result, which is lower because of the higher energy of the mode. Any screening will ultimately reduce the loss as the phonon potentials will become totally screened for zero radius. It can be seen, however, that the DC phonon results are much like the bulk GaAs phonon results whether screening and the effects of coupling are included or not.

\section{SUMMARY}

In this paper, we have examined the momentum relaxation rates and power loss for a Q1D electron gas due to coupling with confined and interface phonon modes. The effects of static and dynamic screening, as well as phonon renormalization are considered. We have found that at low temperatures the coupling between the electron gas and the phonons is important and cannot be ignored in one dimension. The momentum relaxation rates show threshold emission of modes at different energies from those that would be found for just confined and interface phonons and the power loss is higher due to the emission of modes with lower energies only present because of the coupling. At high temperatures the effects of coupling can be ignored, but the scattering by plasmons should be included for momentum relaxation since these add significantly to the phonon scattering. The screening of the phonons only marginally reduces the power loss at high temperatures. Finally we observe that the approximate sum rule for the DC model appears to hold again with screening included.

\section{ACKNOWLEDGMENTS}

We would like to thank B. K. Ridley, M. Babiker, and N. C. Constantinou for useful discussions. This work is supported by the British Council through its Academic Link Scheme. The financial support of EPSRC is gratefully acknowledged. Partial support by the Scientific and Technical Research Council of Turkey (TUBITAK) under Grant No. TBAG-AY/77 is also acknowledged (B.T.). 
${ }^{1}$ D. N. Mirlin and V. I. Perel, Spectroscopy of Nonequilibrium Electrons and Phonons, edited by C. V. Shank and B. P. Zakharchenya (North-Holland, Amsterdam, 1992); Hot Carriers in Semiconductor Nanostructures, edited by J. Shah (Academic, Boston, 1991).

${ }^{2}$ Physics of Hot Electron Transport in Semiconductors, edited by C. S. Ting (World Scientific, Singapore, 1992); S. Das Sarma, J. K. Jain, and R. Jalabert, Phys. Rev. B 41, 3561 (1990).

${ }^{3}$ V. B. Campos and S. Das Sarma, Phys. Rev. B 45, 3898 (1992); S. Das Sarma and V. B. Campos, ibid. 47, 3728 (1993).

${ }^{4}$ X. F. Wang and X. L. Lei, Phys. Status Solidi B 175, 433 (1993); Phys. Rev. B 47, 16612 (1993).

${ }^{5}$ L. Zheng and S. Das Sarma, Phys. Rev. B 54, 2751 (1996).

${ }^{6}$ A. K. Sood, J. Menendez, M. Cardona, and K. Ploog, Phys. Rev. Lett. 54, 2111 (1985).

${ }^{7}$ M. A. Stroscio, Phys. Rev. B 40, 6428 (1989).

${ }^{8}$ M. V. Klein, IEEE J. Quantum Electron. 22, 1760 (1986).

${ }^{9}$ R. Enderlein, Phys. Rev. B 47, 2162 (1993).

${ }^{10}$ N. Mori and T. Ando, Phys. Rev. B 40, 6175 (1989).

${ }^{11}$ K. T. Tsen, K. R. Wald, T. Ruf, P. Y. Yu, and H. Morkoç, Phys. Rev. Lett. 67, 2557 (1991); E. Öztürk, N. C. Constantinou, A. Straw, N. Balkan, B. K. Ridley, D. A. Ritchie, E. H. Linfield, A. C. Churchill, and G. A. C. Jones, Semicond. Sci. Technol. 9, 782 (1994).

${ }^{12}$ B. Tanatar, K. Güven, C. R. Bennett, and N. C. Constantinou, Phys. Rev. B 53, 10866 (1996).

${ }^{13}$ V. B. Campos, S. Das Sarma, and M. A. Stroscio, Phys. Rev. B 46, 38849 (1992); S. Das Sarma, V. B. Campos, M. A. Stroscio, and K. W. Kim, Semicond. Sci. Technol. 7, 860 (1992).
${ }^{14}$ A. C. Maciel, C. Kiener, L. Rota, J. F. Ryan, U. Marti, D. Martin, F. Morier-Gemoud, and F. K. Reinhart, Appl. Phys. Lett. 66, 3039 (1995).

${ }^{15}$ A. R. Goñi, A. Pinczuk, J. S. Weiner, J. M. Calleja, B. S. Dennis, L. N. Pfeiffer, and K. W. West, Phys. Rev. Lett. 67, 3298 (1991); A. Schmeller, A. R. Goñi, A. Pinczuk, J. S. Reiner, J. M. Calleja, B. S. Dennis, L. N. Pfeiffer, and K. W. West, Phys. Rev. B 49, 14778 (1994).

${ }^{16}$ J. K. Jain, R. Jalabert, and S. Das Sarma, Phys. Rev. Lett. 60, 353 (1988); J. K. Jain and S. Das Sarma, ibid. 62, 2305 (1989).

${ }^{17}$ P. M. Platzman and P. A. Wolff, Waves and Interactions in Solid State Plasmas, Solid State Physics, Supplement 13 (Academic Press, New York, 1973); D. Pines and P. Nozieres, The Theory of Quantum Liquids (W. A. Benjamin, New York, 1966).

${ }^{18}$ M. W. C. Dharma-wardana, Phys. Rev. Lett. 66, 197 (1991); 67, 2917 (1991); X. L. Lei and M. W. Wu, Phys. Rev. B 47, 13338 (1993); Z. C. Tao, C. S. Ting, and M. Singh, Phys. Rev. Lett. 70, 2467 (1993).

${ }^{19}$ A. Gold and A. Ghazali, Phys. Rev. B 41, 7626 (1990).

${ }^{20}$ X. F. Wang and X. L. Lei, Phys. Rev. B 49, 4780 (1994); P. E. Selbmann and R. Enderlein, Superlatt. Microstruct. 12, 219 (1992).

${ }^{21}$ C. R. Bennett, N. C. Constantinou, M. Babiker, and B. K. Ridley, J. Phys. Condens. Matter 7, 9819 (1995).

${ }^{22}$ E. H. Hwang and S. Das Sarma, Phys. Rev. B 52, 8668 (1995); C. R. Bennett, N. C. Constantinou, and B. Tanatar, J. Phys. Condens. Matter. 7, L669 (1995).

${ }^{23}$ S. Adachi, J. Appl. Phys. 58, R1 (1985). 